\title{
Bullous pemphigoid associated with milia, increased serum IgE, autoantibodies against desmogleins, and refractory treatment in a young patient*
}

\author{
Shu Ding ${ }^{1}$ \\ Yaping Xiang ${ }^{1}$ \\ Jinhua Huang ${ }^{1}$
}

\author{
Qiancheng Deng ${ }^{1}$ \\ Jing Chen ${ }^{1}$ \\ Jianyun $\mathrm{Lu}^{1}$
}

DOI: http:/ / dx.doi.org/10.1590/abd1806-4841.20176124

\begin{abstract}
Bullous pemphigoid is a blistering autoimmune disease characterized by two hemidesmosomal proteins (anti-BP180 and 230). Pemphigus, by contrast, is characterized by two autoantibodies (anti-desmoglein 1 and 3). Coexistence of autoantibodies of bullous pemphigoid and pemphigus in a patient is rare. A 25-year-old male patient was admitted to our hospital, reporting a 3-month history of multiple papules, vesicles, and erosions over an extensive erythema on the entire body. Laboratory tests showed high levels of serum IgE, anti-BP180 antibodies, and anti-desmoglein 1 and 3. Histopathologic and immunopathologic features were characterized by bullous pemphigoid. No improvement was seen with systemic corticosteroid therapy, however, pulse corticosteriod therapy combined with methylprednisolone, immunosuppressants, immunomodulators, and plasmapheresis led to the recovery of his condition with numerous milia.
\end{abstract}

Keywords: Desmoglein 1; Miliaria; Pemphigoid, bullous; Pemphigus

\section{INTRODUCTION}

Bullous pemphigoid (BP) is an autoimmune subepidermal blistering dermatosis, characterized by an autoimmune response towards two hemidesmosomal proteins within the basement membrane zone (BMZ), namely, BP180 and BP230. ${ }^{1}$ It primarily affects people older than 70 years of age, with no ethnic, racial, or sexual predilection, and rarely occurs in individuals younger than 60 years of age. ${ }^{2}$ Pemphigus is characterized by autoantibodies, namely anti-desmoglein (Dsg) 1 and 3, directed against epidermal surface proteins, leading to acantholysis, which is the loss of cell-to cell adhesion resulting in intraepidermal separation. The diagnosis of autoimmune blistering diseases is frequently confirmed by histological and immunopathological studies. ${ }^{3}$ Here, we report a young patient diagnosed with BP by clinical, histopathological, and immunofluorescence examinations, in which only the anti-BP180 levels were high. Interestingly, autoantibodies proved to be positive for anti-Dsg 1 and 3, and numerous milia appeared during recovery.

\section{CASE REPORT}

A 25-year-old male patient was referred to the dermatology department complaining of multiple papules, vesicles, and erosions over an extensive erythema on the entire body for the last 3 months with no history of allergy and drug intake in the previous months.

Physical examination showed normal vital signs, except for low-grade fever. On the skin, multiple papules, vesicles, tense blisters with serous content and different sizes, erosions and crusts, and an extensive erythema were observed on the entire body (Figure 1). In addition, a giant decubitus ulcer appeared on sacrococcygeal area (Figure 2). Oral mucosa was involved. Nikolsky's sign was negative.

Laboratory tests revealed a significant increase in white blood cell count (19 000/ul) and absolute neutrophil count (13 680/ ul), mild anemia (hemoglobin $90 \mathrm{~g} / \mathrm{l}$ ), hypocalcemia (calcium 1.52 $\mathrm{mmol} / \mathrm{l}$ ), and hypoproteinemia (serum albumin $12.5 \mathrm{~g} / \mathrm{l}$ ). Serum immunoglobulin (Ig) E was $2134 \mathrm{IU} / \mathrm{ml}$ (normal: 0-358 IU/ml), while complement 3 (C3) was 0.67g/1 (normal: 0.75-1.35g/1). A high

\footnotetext{
Work submitted on 05.06.2016

Approved by the Advisory Board and accepted for publication on 26.12.2016

*Work performed at the Department of Dermatology, Third Xiangya Hospital, Central South University, Changsha, China. Financial support: None

Conflict of interest: None

1 Department of Dermatology, Third Xiangya Hospital, Central South University, Changsha, China.
} 
C-reactive protein $(>5 \mathrm{mg} / \mathrm{L})$ and an erythrocyte sedimentation rate (ESR) $(28 \mathrm{~mm} / \mathrm{hr}$ ) were also observed. Autoantibodies were positive for anti-Dsg 1 (138.95U/ml), anti-Dsg 3 (> 150U/ml), and anti-BP180 (> 150U/ml), but proved to be negative for anti-BP230 measured by ELISA (MBL, Japan).

A skin biopsy taken from a bullous lesion showed subepidermal blisters and infiltration of numerous eosinophils in the upper dermis (Figure 3). Direct immunofluorescence (DIF) detected the presence of linear IgG and C3 deposits along the subepidermal basal membrane zone (Figure 4). On Indirect immunofluorescence (IIF), the serum of the patients was positive for anti-BMZ antibodies. IIF-salt split technique showed IgG reactivity with the epidermal side of the BMZ.

The patient's skin condition and health worsened during the first week of treatment with intravenous dexamethasone at 10 $\mathrm{mg}$ /day and cefminox at $2 \mathrm{~g}$ twice/day, followed by therapy adjusted to methylprednisolone at $80 \mathrm{mg} /$ day. Intravenous immunoglobulin (IVIG) therapy ( $20 \mathrm{~g} /$ day) for 5 consecutive days, methotrexate (25 mg/week), and cyclosporine (75 mg twice/day) was added, without improvement of blister formation. After two plasmapheresis sessions, the lesions showed remarkable improvement, but the patient developed new slight blisters. The initial dose of methylprednisolone at $80 \mathrm{mg} /$ day was tapered by $4 \mathrm{mg}$ per week on alternate days to the lowest dose that prevents relapse.

Ten days later, the patient's disease relapsed and deteriorated. Intravenous methylprednisolone at $60 \mathrm{mg} /$ day with IVIG (20 g/ day) for 4 consecutive days was started. Due to the persistent ap- pearance of new blisters, plasmapheresis therapy was again applied to the patient. Minocycline (300 mg twice/day), nicotinamide (150 $\mathrm{mg}$ twice/day), and cyclosporine (75 $\mathrm{mg}$ twice/day) were added. The patient continued to develop new slight blisters. Antibodies of anti-Dsg3 and anti-BP180 were still more than $150 \mathrm{U} / \mathrm{ml}$. Pulse corticosteroid therapy with methylprednisolone (500 mg/day) for five consecutive days was performed with significant improvement in his condition. The anti-Dsg3 titer returned to normal and anti-BP180 was still more than 150. Thereafter, i.v. methylprednisolone and subsequent oral methylprednisolone were tapered gradually. At six months of follow-up, no relapse was observed and a resolution of his condition was seen, with an appearance of numerous milia left on the skin and the healing of the decubitus ulcer (Figures 1 and 2).

\section{DISCUSSION}

Coexistence of autoantibodies of BP and pemphigus in a single patient is quite rare. Patients diagnosed initially as BP and subsequently demonstrating antibodies to both $\mathrm{BP}$ and pemphigus antigens presented a major histocompatibility complex (MHC) II gene HLA-DQb1*0301, which was typically found in patients with BP. Moreover, many of those patients also carry the MHC II genes typically correlated with pemphigus patients. ${ }^{4}$ However, autoantibodies of pemphigus and BP may also appear in healthy people. ${ }^{5}$

Noticeably, a high level of serum IgE was found in this patient. IgE may well contribute to the pathogenicity of BP. Direct proof derives from studies of human skin xenografts to mice. ${ }^{1}$ The total serum IgE level correlates closely with disease activity as well as with
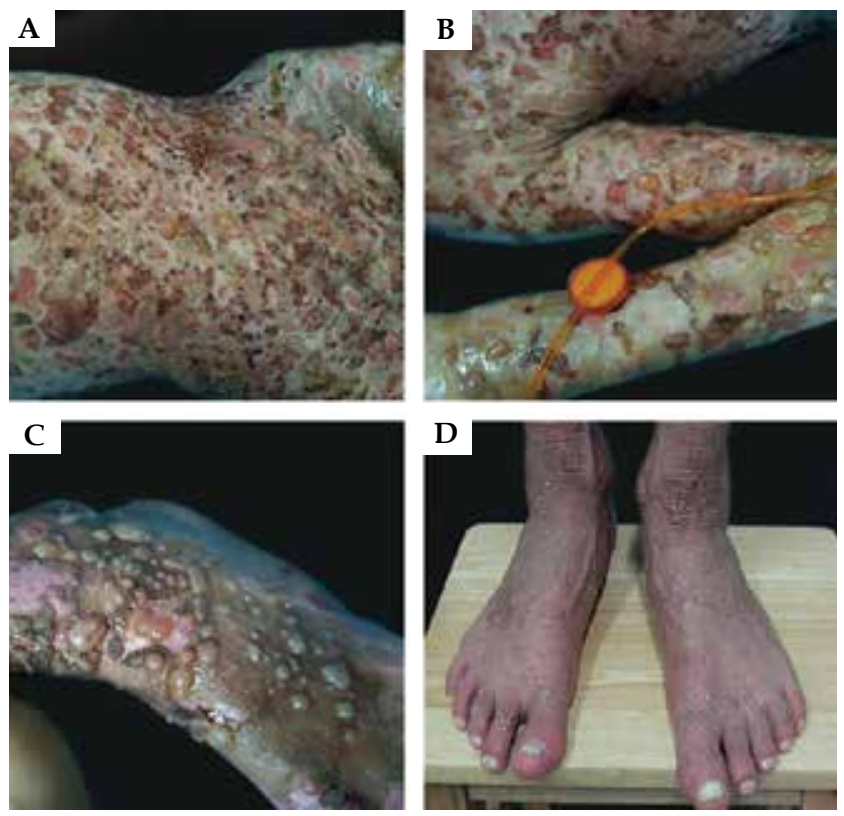

Figure 1: A-C: Numerous erythemas with papules, vesicles, tense blisters, erosions, and crusts were observed on the entire body.

D: Numerous milia during recovery
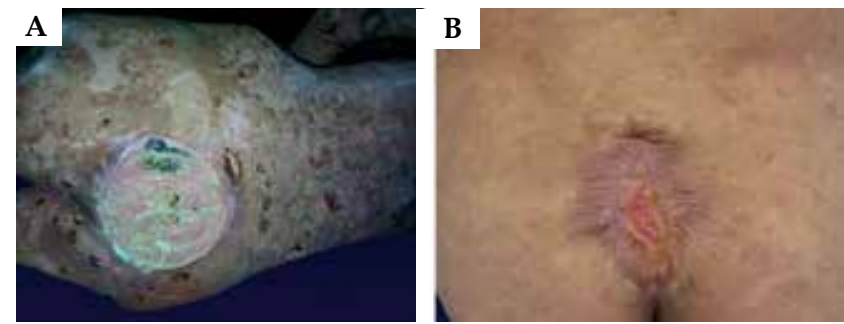

Figure 2: A - A giant decubitus ulcer was presented on sacrococcygeal area. B - Decubitus ulcer during recovery
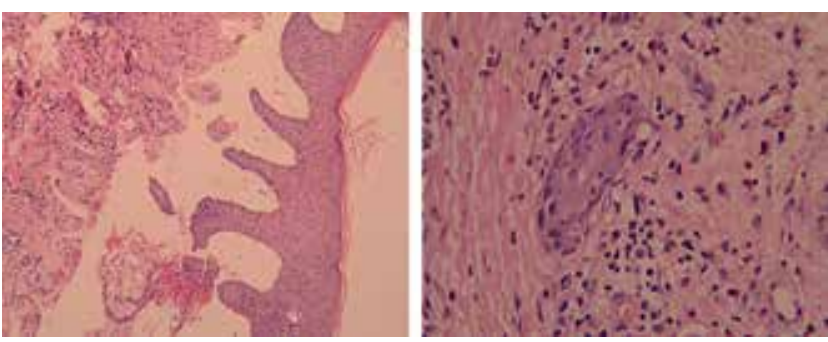

Figure 3: Histopathology of the skin lesion revealed subepidermal blisters (Hematoxylin \& eosin, X10) and infiltration of numerous eosinophils in the upper dermis (Hematoxylin \& eosin, X40) 


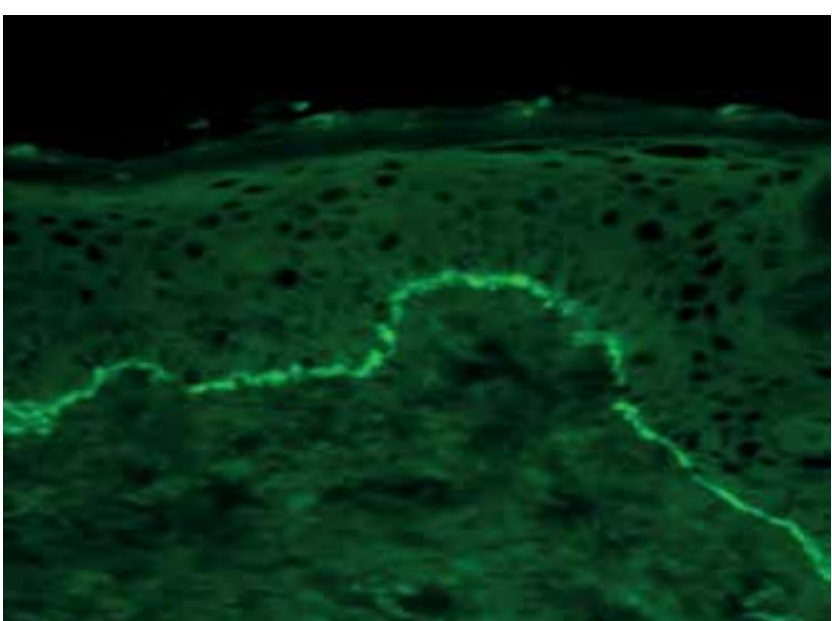

Figure 4: Direct immunofluorescence showed linear IgG and C3 deposits along the subepidermal basal membrane zone
anti-BMZ antibodies in BP. ${ }^{6}$ Diagnosis of BP is based on a combination of clinical, histopathological, and immunofluorescence exams, which can also be observed in epidermolysis bullosa acquisita (EBA), another subepidermal bullous disease. ${ }^{3}$ However, the IF-salt split technique can be used to differentiate them. In BP, IgG deposits can be found on either the epidermal or the dermal side, rarely on both sides, while EBA deposits only occur on the dermal side., ${ }^{3,7}$

Systemic corticosteroid therapy seems to be the best established initial treatment for BP. High doses of systemic corticosteroids and immunosuppressants were applied to this patient with satisfactory response. After combined therapy of pulse corticosteroid therapy, antibiotics, immunosuppressants, IVIG, and plasmapheresis, the patient's condition improved greatly. It is possible that the presence of two potentially pathogenic autoantibodies contributed to treatment failure in this case. ${ }^{8}$

This report was also attracted to the formation of milia during recovery, which seems to occasionally occur in BP. ${ }^{9}$ Immunological predisposition, aberrant interaction between the hemidesmosomes, and the extracellular matrix components beneath the hemidesmosomes have been described as regards the formation of numerous milia during recovery. ${ }^{10}$

\section{REFERENCES}

1. Hammers CM, Stanley JR. Mechanisms of Disease: Pemphigus and Bullous Pemphigoid. Annu Rev Pathol. 2016;11:175-97.

2. Zanella RR, Xavier TA, Tebcherani AJ, Aoki V, Sanchez AP. Bullous pemphigoid in younger adults: three case reports. An Bras Dermatol. 2011;86:355-8.

3. Reis-Filho EG, Silva Tde A, Aguirre LH, Reis CM. Bullous pemphigoid in a 3-monthold infant: case report and literature review of this dermatosis in childhood. An Bras Dermatol. 2013;88:961-5.

4. Zakka LR, Keskin DB, Reche P, Ahmed AR. Relationship between target antigens and major histocompatibility complex (MHC) class II genes in producing two pathogenic antibodies simultaneously. Clin Exp Immunol. 2010;162:224-36.

5. Prüßmann W, Prüßmann J, Koga H, Recke A, Iwata H, Juhl D, et al. Prevalence of pemphigus and pemphigoid autoantibodies in the general population. Orphanet $J$ Rare Dis. 2015;10:63.

6. van Beek N, Schulze FS, Zillikens D, Schmidt E. IgE-mediated mechanisms in bullous pemphigoid and other autoimmune bullous diseases. Expert Rev Clin Immunol. 2016;12:267-77.

7. Delaporte E, Piette F, Janin A, Cozzani E, Joly P, Thomine E, et al. Pemphigoid mimicking epidermolysis bullosa acquisita. Ann Dermatol Venereol. 1995;122:19-22.

8. Sami N, Bhol KC, Beutner EH, Plunkett RW, Leiferman KM, Ahmed AR. Diagnostic features of pemphigus vulgaris in patients with bullous pemphigoid. Molecular analysis of autoantibody profile. Dermatology. 2002;204:108-17.

9. Tsuruta D, Brzezinski P, Koga H, Ohata C, Furumura M, Hashimoto T. Bullous pemphigoid with prominent milium formation. Acta Dermatovenerol Croat. 2013:21:35-8.

10. Uchida S, Oiso N, Koga H, Ishii N, Okahashi K, Matsuda H, et al. Refractory bullous pemphigoid leaving numerous milia during recovery. J Dermatol. 2014;41:1003-5.
MAILING ADDRESS:

Jianyun Lu

Department of Dermatology,

Third Xiangya Hospital, Central South University,

No. 138, Tongzipo Road,

Changsha

410013 Hunan, China.

E-mail: 1010737024@qq.com

How to cite this article: Ding S, Deng QC, Xiang YP, Chen J, Huang JH , Lu JY. Bullous pemphigoid associated with milia, increased serum IgE, autoantibodies against desmogleins, and refractory treatment in a young patient. An Bras Dermatol. 2017;92(5 Suppl 1): 34-6. 